\title{
Quantitative measurements of myocardial perfusion and function from SPECT (and PET) studies depend on the method used to perform those measurements
}

\author{
Guido Germano, PhD ${ }^{\mathrm{a}, \mathrm{b}}$ \\ a Cedars-Sinai Medical Center, UCLA, Los Angeles, CA \\ b David Geffen School of Medicine, UCLA, Los Angeles, CA
}

Received Dec 6, 2016; accepted Dec 7, 2016

doi: 10.1007/s12350-016-0757-z

\section{See related article, pp. 911 -924}

One would perhaps be justified to remark that the title of this editorial only states the obvious and that its premise ought to be accepted by any reasonable reader, even if not particularly familiar with nuclear cardiology. Nevertheless, the paper to which the editorial refers, ${ }^{1}$ describing good concordance but also substantial differences amongst three commercially available software packages to quantify LV perfusion and function from gated myocardial SPECT, offers us the opportunity to reflect on some of the specific reasons why those differences exist. As the authors describe in their comprehensive review of the published literature, a wealth of studies exists assessing the cross-correlation of the outputs of different quantitative gated SPECT algorithms, and the current study confirms previously reported findings, albeit in a larger population of 634 consecutive patients. Let us try to interpret and clarify those findings, while framing them in a more general context.

\section{DIFFERENT ALGORITHMS ARE BASED ON DIFFERENT COMPUTATIONAL TOOLS (AND WORKING ASSUMPTIONS)}

The specific software packages used by Alexiou et al are Emory University's Cardiac Toolbox (ECTb), ${ }^{2}$

Reprint requests: Guido Germano, PhD, Cedars-Sinai Medical Center, UCLA, Los Angeles, CA, 90095, USA; Guido.Germano@cshs.org J Nucl Cardiol 2018;25:925-8.

$1071-3581 / \$ 34.00$

Copyright (c) 2016 American Society of Nuclear Cardiology.
Cedars-Sinai's Cardiac Suite (QGS/QPS), ${ }^{3}$ and General Electric's Myovation. This writer is not aware of any published papers detailing the technical principles on which the Myovation software is based, but the other two algorithms and their differing characteristics have been well described in the literature. As far as measurement of global function is concerned, the ECTb performs a Fourier decomposition of the time-volume (T-V) curve that describes the LV cavity volume as a function of the cardiac cycle, then takes the first Fourier harmonic to estimate end-systolic (ES) and end-diastolic (ED) volumes - as a result, $\mathrm{T}-\mathrm{V}$ curve estimates are less dependent on temporal sampling, and ejection fractions (EFs), EDVs and ESVs from 8-frame and 16-frame gated acquisitions will be relatively close in value. ${ }^{2}$ In contrast, QGS/QPS measures the actual dynamic range of the T-V curve, which is directly proportional to the frequency of temporal sampling-in other words, 8frame ESVs will be larger, EDVs smaller, and EFs lower compared to 16-frame gated acquisitions, with a substantially uniform difference of 3-4 EF points over the entire EF range. ${ }^{4}$ With regard to myocardial thickening, the ECTb algorithm assumes a uniform myocardial thickness of $1 \mathrm{~cm}$ at ED, and increases it with a 1:1 relationship to the apparent myocardial "brightening", from ED to ES, since partial volume effect is fairly linear for the reconstructed spatial resolutions and myocardial thicknesses of interest. ${ }^{2}$ QGS/QPS is also based, in part, on myocardial brightening, but uses phantom calibration to estimate ED thickness, and asymmetrical Gaussian fitting with a constant myocardial mass constraint to follow the endocardium and epicardium's location throughout the cardiac cycle. ${ }^{3}$ As for perfusion quantification, aside from the different myocardial sampling techniques (hybrid cylindri$\mathrm{cal} /$ spherical for ECTb, ellipsoidal for QGS/QPS), the 
ECTb generates normal databases using three patient populations (a normal group with low likelihood of coronary artery disease (CAD), a criteria group with a spectrum of perfusion abnormalities, and a prospective validation group), ${ }^{2}$ whereas QGS/QPS employs a simplified approach based on a single patient population with low likelihood of CAD, in which normal limits are derived without visual scoring of abnormal scans or optimization of regional thresholds. ${ }^{5}$ Without elaborating further, one should expect different algorithms to produce somewhat different quantitative results.

\section{DIFFERENT ALGORITHMS MAY BE AUTOMATED TO DIFFERENT DEGREES}

While most available quantitative software packages are largely automated and capable of generating quantitative outputs at the push of a button, that is not always the case. For example, Myovation does not provide standardized segmental perfusion scores, but rather the average segmental count values relative to maximum pixel values in the relevant polar plot, which the authors converted to categorical scores according to $>70 \%, 50-69 \%, 30-49 \%, 10-29 \%$ and $<10 \%$ thresholds. ${ }^{6}$ Likewise, seed points, masking information, or outright location of myocardial structures may need to be provided as inputs to the software.

Even when algorithms are completely automated, sometimes the myocardial contours they detect (from which quantitative measurements are performed) need to be corrected-this happens particularly in connection with the valve plane's location, ${ }^{8}$ or, less frequently, if LV segmentation fails altogether due to excessive extracardiac activity. ${ }^{9}$ Fallback mechanisms are typically provided to manually override automatically produced contours-but when manual interaction is required, results will necessarily differ, both between algorithms and intra-algorithm.

\section{GOOD CROSS-CORRELATION DOES NOT MEAN INTERCHANGEABILITY OF RESULTS}

The different principles and assumptions on which different algorithms are based, potentially compounded by imperfect automation and human operator interaction, make it indeed remarkable that correlation of quantitative outputs produced from different software packages in the same patient populations has been reported to be quite good. ${ }^{7}$ This is especially true for Spearman's Rank correlation coefficient (the slope of the correlation line), often well in excess of 0.9 in crosssoftware comparisons; the intercept of the correlation line, however, is typically different from zero, suggesting systematic bias, as confirmed by Bland-Altman analyses. As the authors show, threshold-based classification of parameters of myocardial perfusion and function highlights discordances related to the choice of software used to quantify those parameters. ${ }^{1}$

The more complex the measurement of a parameter (usually because derived from other parameters), the greater the propagation of measuring errors/biases and the lower the agreement between different techniques to measure the derived parameter (as demonstrated, for example, by the quantification of myocardial flow reserve (MFR) from 82Rb PET using 10 different software approaches in the RUBY-10 Study). ${ }^{10}$ The take-home message: quantitative outputs of different algorithms generally correlate well, but are not interchangeable.

\section{WHAT IS THE “TRUE” VALUE OF A CARDIAC PARAMETER?}

Since software-related differences in quantitative measurements do exist, it is natural to wonder whether one approach is more accurate than others in estimating the true value of a parameter, as determined by an appropriate "gold standard." Most myocardial perfusion SPECT and PET quantitative software packages have been validated using a variety of reference techniques, some with superior spatial resolution (MRI), others with high temporal resolution (first pass, contrast ventriculography, blood pool SPECT). ${ }^{7}$ Unfortunately, however, those theoretical gold standards have their own limitations, a most notable one being that their measurements are almost invariably less automated than those they seek to validate, and consequently more dependent on human operation and more susceptible to error. In part because of this, it has been noted that, for example, the average LVEF values calculated in patient populations with low likelihood of coronary disease vary according to the modality employed to measure them (Figure 1). ${ }^{11}$

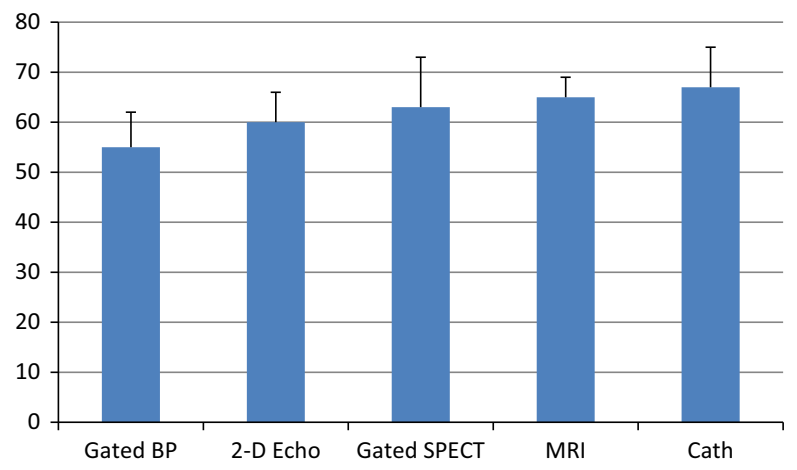

Figure 1. Published "normal values" of LVEF as a function of the modality employed to perform the imaging study (courtesy of Kenneth Nichols, PhD). 
Additionally, there are some known limitations of nuclear cardiology that make it intrinsically difficult, for any software, to measure the true value of parameters in certain groups of patients. For example, limited spatial resolution leads to the overestimation of LVEFs in patients with small LVs, while the assumptions and calibrations used to estimate myocardial thickness do not practically apply to patients with left ventricular hypertrophy (LVH). That notwithstanding, nuclear cardiology software does a very good job at dealing with the great majority of the typically imaged patients.

Because it has been already established by many published reports (including the current one) that systematic differences exist amongst algorithms, a working hypothesis is that their measurements, if repeated on the same image dataset and for the same parameter of interest, will cluster in the proximity of the "real" value but not necessarily overlap with it, as shown in Figure 2. As we will argue, whether one cluster is closer than the others to the bullseye's center (accuracy) is not as relevant to patient management and clinical outcome as one might think; conversely, it is essential that a cluster be tight (repeatability).

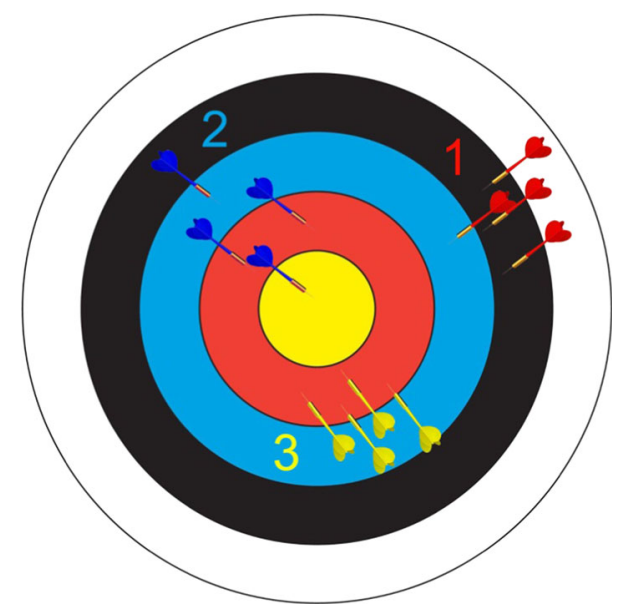

Figure 2. Conceptual representation of three theoretical software packages (not the ones in Alexiou's report) quantifying repeatedly (four times in a row) a specific cardiac parameter from a single SPECT or PET image dataset. If the center of the bullseye symbolizes the "true" value of that parameter, different algorithms may achieve different degrees of closeness to it (accuracy). More important, however, is how tightly clustered the repeated measurements by the same software are (repeatability). In this example, algorithm 2 achieved the highest accuracy in one measurement, but it was overall less consistent, or its measurements less repeatable.

\section{REPEATABILITY MAY BE CLINICALLY MORE IMPORTANT THAN ACCURACY}

Accuracy of nuclear cardiac quantification, while theoretically attainable, cannot be conclusively verified based on currently available gold standards. On the other hand, risk assessment and stratification of patients with suspected or known CAD is based in substantial part on quantitative measurements, with patient management increasingly focused on reducing potentially preventable adverse events such as cardiac death, nonfatal myocardial infarction, or CAD progression. For example, a 10,627-patient observational study showed that medical therapy results in increased survival versus revascularization if no or mild stress/rest ischemia $(<10 \%$ of the total myocardium) is measured by SPECT, ${ }^{12}$ while another 6,713-patient study showed that gender-specific quantitative thresholds for $\mathrm{EF}$ and ESV provide incremental prognostic information over perfusion, when cardiac death and hard events are concerned. ${ }^{13}$

Because quantitative measurements are algorithmspecific, it is important that the appropriate risk stratification and prognostic thresholds for the software of interest be used, based on published data for the related study protocol. It is also essential that a high degree of confidence in the repeatability of the quantitative measurements exist. This means, at a minimum, verifying that repeated applications of an algorithm to the same dataset produce similar results (a tight cluster in Figure 2, with ideally perfect superposition if the algorithm is fully automated). ${ }^{14}$ In the future, we should expect all software to become more "intelligent," for example by incorporating knowledge of the fact that images from different studies may belong to a same patient, even if acquired at different times and under different protocols. ${ }^{15}$

\section{PRACTICAL CONCLUSION}

From this editorialist's experience, if the quantitative software you own has been validated for the type of imaging study you perform and a patient population reasonably similar to yours, use it with confidence that it will produce reliable results. Particularly when comparison of quantitative results is the goal (for example, with serial studies), try to minimize any protocol-related variabilities: if at all possible, perform the repeat study on the same camera, using the same radioisotope, dose, acquisition, and processing protocol. As done for the acquisition and processing chain, use the same software to analyze and quantify serial studies, without mixingand-matching outputs of different algorithms. 


\section{References}

1. Alexiou S, Georgoulias P, Angelidis G, Valotassiou V, Tsougos I, Psimadas D, et al. Myocardial perfusion and left ventricular quantitative parameters obtained using gated myocardial SPECT: comparison of three software packages. J Nucl Cardiol. 2016. doi: 10.1007/s12350-016-0730-x.

2. Garcia EV, Faber TL, Cooke CD, Folks RD, Chen J, Santana C. The increasing role of quantification in clinical nuclear cardiology: The Emory approach. J Nucl Cardiol. 2007;14:420-32.

3. Germano G, Kavanagh PB, Slomka PJ, Van Kriekinge SD, Pollard G, Berman DS. Quantitation in gated perfusion SPECT imaging: The Cedars-Sinai approach. J Nucl Cardiol. 2007;14:433-54.

4. Germano G, Kiat H, Kavanagh PB, Moriel M, Mazzanti M, Su HT, et al. Automatic quantification of ejection fraction from gated myocardial perfusion SPECT. J Nucl Med. 1995;36:2138-47.

5. Slomka PJ, Nishina H, Berman DS, Akincioglu C, Abidov A, Friedman J, et al. Automated quantification of myocardial perfusion SPECT using simplified normal limits. J Nucl Cardiol. 2005;12:66-77.

6. Alexiou, S. Personal communication.

7. Germano G, Berman D. Gated single-photon emission computed tomography. In: Iskandrian AE, Garcia EV, editors. Nuclear cardiac imaging: principles and applications. New York: Oxford University Press; 2008. p. 161-83.

8. Xu Y, Kavanagh P, Fish M, Gerlach J, Ramesh A, Lemley M, et al. Automated quality control for segmentation of myocardial perfusion SPECT. J Nucl Med. 2009;50:1418-26.
9. Germano G, Kavanagh PB, Fish MB, Lemley MH, Xu Y, Berman DS, Slomka PJ. "Same-patient processing" for multiple cardiac studies. 1. Improving LV segmentation accuracy. J Nucl Cardiol. 2016;23(6):1435-41.

10. Nesterov SV, Deshayes E, Sciagra R, Settimo L, Declerck JM, Pan $\mathrm{XB}$, et al. Quantification of myocardial blood flow in absolute terms using Rb-82 PET imaging the RUBY-10 study. JACCCardiovasc Imag. 2014;7:1119-27.

11. Nichols, K. Personal communication.

12. Hachamovitch R, Hayes SW, Friedman JD, Cohen I, Berman DS. Comparison of the short-term survival benefit associated with revascularization compared with medical therapy in patients with no prior coronary artery disease undergoing stress myocardial perfusion single photon emission computed tomography. Circulation. 2003;107:2900-7.

13. Sharir T, Kang XP, Germano G, Bax JJ, Shaw LJ, Gransar H, et al. Prognostic value of poststress left ventricular volume and ejection fraction by gated myocardial perfusion SPECT in women and men: Gender-related differences in normal limits and outcomes. J Nucl Cardiol. 2006;13:495-506.

14. Germano G, Kavanagh PB, Slomka PJ, Berman DS. Tracking a therapeutic response: How reliable are serial measurements of LV perfusion and function? J Nucl Cardiol. 2012;19:360-3.

15. Germano G, Kavanagh PB, Ruddy TD, Wells RG, Xu Y, Berman DS, Slomka PJ. "Same-patient processing" for multiple cardiac studies. 2. Improving quantification repeatability. J Nucl Cardiol. 2016;23(6):1442-53. 